\title{
ANALYTICAL INVESTIGATIONS OF TEMPERATURE EFFECTS ON CREEP STRAIN RELAXATION OF BIOMATERIALS USING HOMOTOPY PERTURBATION AND DIFFERENTIAL TRANSFORM METHODS
}

\author{
Olurotimi Adeleye, Olakanla Abdulkareem \\ Department of Systems Engineering, University of Lagos \\ Akoka, Lagos, Nigeria \\ rotimiadeleye1711@gmail.com, olaks1@live.com \\ Ahmed Yinusa and GBeminiyi Sobamowo \\ Department of Mechanical Engineering, University of Lagos, \\ Akoka, Lagos, Nigeria \\ mynotebook2010@yahoo.com, mikegbeminiyi@gmail.com
}

[Received: February 14, 2019; Accepted: August 22, 2019]

\begin{abstract}
In this paper, the effect of temperature on relaxation of creep strain in biomaterials is modeled and analyzed with homotopy perturbation and differential transform methods. Polymeric biomaterials used as implants undergo both geometric and material nonlinear deformation when subjected to different loading conditions. The present study is concerned with the effects of temperature on the geometric nonlinear deformation of the relaxation of creep strain in these materials. Polymeric biomaterials exhibit time dependent response as observed in viscoelastic materials and this is represented by a one-dimensional rheological material model with constant material parameters. This model is then extended to capture the effects of temperature and the resulting final governing model is a nonlinear differential equation which cannot be easily solved by the standard analytic techniques. Here, two efficient special nonlinear analytic techniques, the homotopy perturbation and differential transform methods, are applied to obtain the solution of the developed nonlinear differential equation. The obtained analytical solutions are validated with the fourth-order Runge-Kutta numerical method. An error analysis shows that good agreement exists between the solutions obtained with these methods. The effects of some parameters on the model were investigated. As observed from the study, it can be shown that an increase in thermal conductivity and viscosity resulted in an increase in resistance to deformation of the material, while an increase in the material stiffness resulted in an increase in the rate of deformation and relaxation.
\end{abstract}

Mathematical Subject Classification: 80A20, 80M25

Keywords: Creep, relaxation, temperature, alginate hydrogel biomaterials, homotopy perturbation method, differential transform method

\section{INTRODUCTION}

Polymers have a wide range of applications such as: automobile, aviation, electronics, and packaging industries. But in the past few decades, polymers have been extended 
to biomedical applications. The polymers used for biomedical applications as a result of their biocompatibility, controllable biodegradation rate and their biofunctional properties, include natural polymers, which are polysaccharides (i.e. starch, glycogen) and synthetic polymers, which are: poly(glycolic acid) (PGA) and poly(hydroxyl butyrate) (PHB) 1. Several devices and implants have been studied and observed throughout the medical field, and they have been developed for various applications in the human body (Figure 1). These ranges from artificial support, such as synthetic blood vessel, hip replacement and knee/joint implants, to applications that alleviate the human organ functions, e.g. pacemaker. These applications also tend to vary according to their positions and placement within the body. The areas of application of these devices include regions with high chemical, electrical and mechanical activity, such as when they are used in regions of high mechanical stress for the replacement of bone. Biomaterials tend to undergo deformation over time especially when temperature around the material increases beyond the normal temperature, hence there is a need to investigate the effects of this increase in temperature on relaxation of creep strain in order to avoid failure of these materials before replacement.

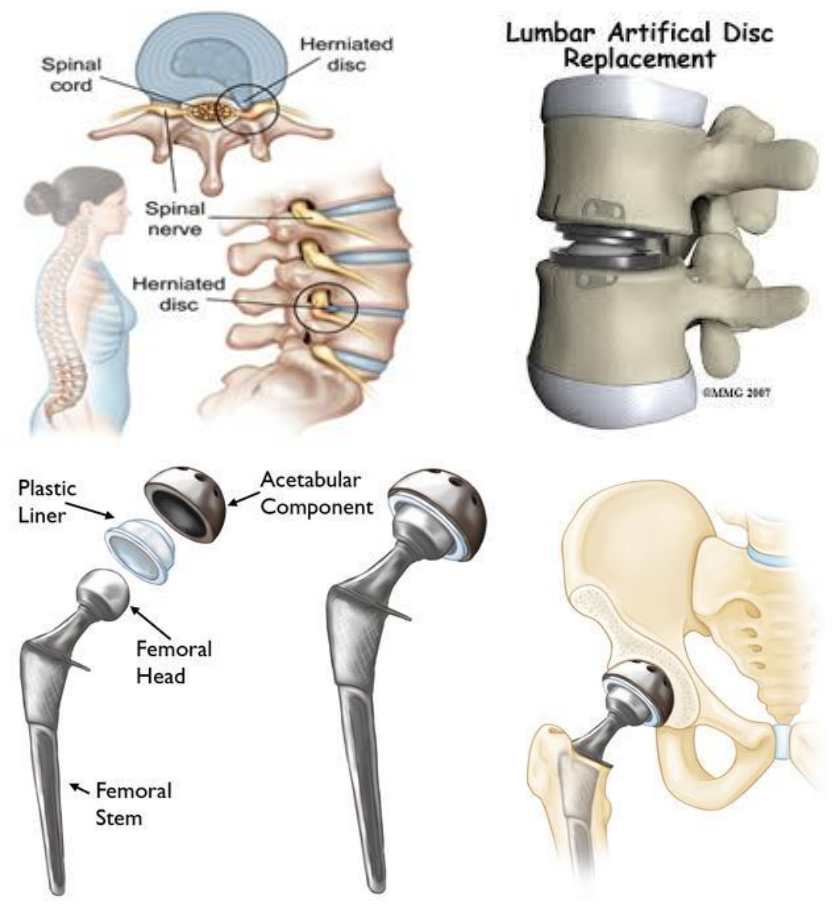

Figure 1. Biomaterial applications: Lumbar artificial disc replacement, Hip liner replacement

Nonlinear behaviors have been observed in the deformation of the biomaterials as shown in the stress-strain curve of an alginate hydrogels 2 which are extremely dependent on the strain rate. This is also noticed in tensile tests of ligaments and tendons 3,4 . Notable successes have been obtained in the use of nonlinear models 
for the behavior of biomaterials 5,6 . It has also been observed that most polymeric biomaterials exhibit viscous tendencies and these behaviors are nonlinear in nature [5]. There are different requirements for the device and implant which are important for the continued existence of the implants and the comfort of the user, which are chemical, electrical, thermal and mechanical characteristics of the substrate for implants and packaging for devices 7 . Stress relaxation tests have often been used as a convenient technique for determining the rheological behaviors of several viscoelastic materials. As a result of differences in material characteristics, several linear and nonlinear viscoelastic models exist which represent the relationship between the time and the stress modulus 810 .

In the present study, a nonlinear viscoelastic model has been developed by using the stress decomposition theory to analyze the effects of temperature in the relaxation of creep strain in biomaterials. The model is represented by a nonlinear differential equation whose solutions cannot be easily obtained with the standard analytical techniques. There are special analytical techniques for solving nonlinear differential equations. Two among them have been used to obtain the solution of the developed nonlinear differential equation; the homotopy perturbation and the differential transform methods. The homotopy perturbation method (HPM) was first proposed by Ji-Huan He [11 for solving integral and differential equations for both linear and nonlinear models. It is a powerful analytical method which does not require any approximation. A number of studies have been carried out to establish the strength of HPM by comparing the results obtained to other methods such as Adomian Decomposition Method (ADM) [12. Remarkably, these studies have concluded that the method is more accurate and effective than ADM. Other studies carried out with the homotopy perturbation method (HPM) include its use for the analysis of heat transfer in longitudinal fins [13], the use of HPM and collocation method (CM) for analysis of thermal performances of porous fin with temperature-dependent heat generation 14 and heat transfer analysis of non-Newtonian natural convective fluid flow using homotopy perturbation and Daftardar-Gejiji and Jafari methods 15].

Another efficient method for determining exact solution in nonlinear phenomena is the differential transform method (DTM). The differential transform method is an approximate analytical method for solving linear and nonlinear differential equations. The differential transformation method (DTM) was developed by Zhou in 1986 [16. 17. It is also used in providing solutions to both linear and nonlinear partial differential equations 18 . It has a lower computational intensity than other approximate methods and the accuracy is higher than those methods [19, 20]. The distinguishing features of DTM have been emphasized in quite a number of studies 21]. Comparisons have been made between the results of DTM and results obtained with other methods such as adomian decomposition method (ADM) [22] and finite element method (FEM) [19]. In order to achieve better results, the efficiency of DTM has been combined with other methods 20]. These methods include the Laplace transform, which was used to overcome the deficiency that emanated from unsatisfied conditions 23 . DTM was used to solve the problems of phagocyte transmigration for foreign body 
responses to subcutaneous biomaterial implantations [24] and creep strain relaxations in biomaterials 25].

Hence, the aim of this study is to investigate the problem of temperature effects on relaxation of creep strain in polymeric biomaterials. This problem, which is governed by a developed nonlinear differential equation, is solved using two efficient methods; the homotopy perturbation method and differential transform method. The fourthorder Runge-Kutta numerical method is then applied to validate the obtained solution from the two methods. The effects of thermal conductivity, viscosity and material stiffness on the deformation of these biomaterials are then considered.

\section{Problem DESCRIPTION}

A nonlinear material model has been adopted due to the viscous nature of the polymeric biomaterials. This non-linear material has elastic, inertial nonlinearities and viscous parameters as presented by Monsia 5]. A logarithm spring force law is used in this model which describes the unloading response of a viscoelastic material perfectly when subjected to constant loading.

$$
\varphi(\varepsilon)=\ln \left(\varepsilon_{0}-\frac{\varepsilon(t)}{\varepsilon_{0}}\right)
$$

$\varepsilon_{0} \neq 0$ is a material constant. As expressed by Monsia [5] the function exists if and only if $\varepsilon_{0}>\sqrt{\varepsilon(t)}$. The function $\varphi(\varepsilon)$ has a vertical asymptote at $\varepsilon(t)=\varepsilon_{0}^{2}$, which implies $\varphi(\varepsilon)$ is not defined for $\varepsilon>\varepsilon_{0}^{2}$. Therefore the nonlinear ordinary differential equation from Bauer's theory [26], by superposing inertial stresses, pure elastic, and viscosity, for a nonlinear spring force function $\varphi(\varepsilon)$ can be rewritten in the form

$$
\begin{gathered}
\frac{a \varphi(\varepsilon)}{c}+\frac{b \dot{\varepsilon} \varphi^{\prime}(\varepsilon)}{c}+\dot{\varepsilon}^{2} \varphi^{\prime \prime}(\varepsilon)+\ddot{\varepsilon} \varphi^{\prime}(\varepsilon)=\frac{\sigma_{t}}{c} \\
\varepsilon(0)=\varepsilon_{i} \quad \dot{\varepsilon}(0)=0
\end{gathered}
$$

where $a$ and $b$ are the viscosity and stiffness time independent coefficients respectively, $c$ is the time independent inertia module, $\varepsilon(t)$ denotes the time dependent strain, $\varepsilon_{i}$ is the initial strain, and $\sigma_{t}$ denotes the total exciting stress acting on the material. The relationship between the deformation temperature parameter, the flow stress (i.e. exciting stress) and strain rate can be represented by hyperbolic sine Arrhenius type equation [27].

$$
|\dot{\varepsilon}|=A F(|\sigma|) \exp [-Q / R T]
$$

where $F(\sigma)$ is a parameter function of stress, $A$ is a material constant, $\dot{\varepsilon}$ is the strain rate, $Q$ is the activation energy, $R$ is the universal gas constant, and $T$ is the absolute temperature. The effects of the temperature and strain rate on the deformation can be denoted by the Zener-Hollomon parameter $Z$ [28]:

$$
Z=|\dot{\varepsilon}| \exp [-Q / R T]
$$

Using equation (4), and substituting the exponential law into equation (3), the strain rate is obtained as

$$
|\dot{\varepsilon}|=A|\sigma|^{n} \exp [-Q / R T]
$$

where $n$ is a material constant. 


\section{SOlution FOR TEMPERATURE EFFECTS ON STRAIN-RATE}

Utilizing equation (1) and (5) equation (2) can be rewritten as

$$
\begin{gathered}
\frac{a \ln \left(\varepsilon_{0}-\varepsilon / \varepsilon_{0}\right)}{b}-\frac{b \dot{\varepsilon}}{c \varepsilon_{0}\left(\varepsilon_{0}-\varepsilon / \varepsilon_{0}\right)}-\frac{\ddot{\varepsilon}}{\varepsilon_{0}\left(\varepsilon_{0}-\varepsilon / \varepsilon_{0}\right)}-\frac{\dot{\varepsilon}^{2}}{\varepsilon_{0}^{2}\left(\varepsilon_{0}-\varepsilon / \varepsilon_{0}\right)}= \\
=\frac{\dot{\varepsilon}}{A c} \exp [Q / R T] .
\end{gathered}
$$

The exponential function $\exp Q / R T$ in equation (5) can be expanded as

$$
e^{Q / R T} \simeq 1+\frac{Q}{R T}+\frac{(Q / R T)^{2}}{2 !} \ldots
$$

Substituting equation (7) into (6) the evolution equation of deformation becomes

$$
\begin{gathered}
\frac{c\left(\varepsilon_{0}^{2}-\varepsilon\right) \ddot{\varepsilon}+b\left(\varepsilon_{0}^{2}-\varepsilon\right) \dot{\varepsilon}+c \dot{\varepsilon}^{2}}{c\left(\varepsilon_{0}^{2}-\varepsilon\right)^{2}}-\frac{\dot{\varepsilon}}{A c}-\frac{\dot{\varepsilon} Q}{A c R T}-\frac{a \ln \left(\varepsilon_{0}-\varepsilon / \varepsilon_{0}\right)}{c}=0 \\
\varepsilon(0)=\varepsilon_{i} \quad \dot{\varepsilon}(0)=0
\end{gathered}
$$

Equation $(8)$ is the nonlinear evolution equation of deformation for material under the effects of temperature.

\section{Determining the time-Deformation equation}

In order to resolve (8), a change of variables is considered. By utilizing the transformation

$$
\varepsilon_{0} e^{x}=\varepsilon_{0}^{2}-\varepsilon, \quad x=\ln \left(\frac{\varepsilon_{0}^{2}-\varepsilon}{\varepsilon_{0}}\right)
$$

for $\dot{\varepsilon}$ and $\ddot{\varepsilon}$ we have $\dot{\varepsilon}=-\dot{x} \varepsilon_{0} e^{x}$ and $\ddot{\varepsilon}=-\ddot{x} \varepsilon_{0} e^{x}-\dot{x}^{2} \varepsilon_{0} e^{x}$. After substituting them into (8) the nonlinear evolution equation of deformation has the form

$$
\begin{aligned}
& \frac{e^{-2 x}\left(b \varepsilon_{0} e^{x}\left(-\dot{x} \varepsilon_{0} e^{x}\right)+c\left(-\dot{x} \varepsilon_{0} e^{x}\right)^{2}+c \varepsilon_{0} e^{x}\left(-\ddot{x} \varepsilon_{0} e^{x}-\dot{x}^{2} \varepsilon_{0} e^{x}\right)\right)}{c\left(\varepsilon_{0}^{2}-\varepsilon\right)^{2}}- \\
&-\frac{a x}{c}+\frac{\dot{x} \varepsilon_{0} e^{x}}{A c}+\frac{\dot{x} \varepsilon_{0} e^{x} Q}{A c R T}=0
\end{aligned}
$$

which can be simplified further to

$$
\frac{-b \dot{x}}{c}+\dot{x}^{2}-\ddot{x}-\dot{x}^{2}-\frac{a x}{c}+\frac{\dot{x} \varepsilon_{0} e^{x}}{A c}\left(1+\frac{Q}{R T}\right)=0 .
$$

Introducing the notations $b / c=\alpha, a / c=\beta$ and $1 /(A c)=\gamma$ equation (11) becomes

$$
\ddot{x}+\alpha \dot{x}+\beta x-\gamma \dot{x} \varepsilon_{0} e^{x}\left(1+\frac{Q}{R T}\right)=0 .
$$

Here $\alpha$ is the viscosity coefficient, $\beta$ is the stiffness coefficient and $\gamma$ is the thermal coefficients, respectively.

Substituting the first four terms of the series $e^{x} \simeq 1+x+\frac{x^{2}}{2 !}+\frac{x^{3}}{3 !}+\ldots$ into 12 yields

$$
\ddot{x}+\alpha \dot{x}+\beta x-\gamma \dot{x} \varepsilon_{0}\left(1+x+\frac{x^{2}}{3}+\frac{x^{3}}{6}\right)\left(1+\frac{Q}{R T}\right)=0 .
$$


Equation 13 can also be expanded and expressed as

$$
\begin{aligned}
& \ddot{x}+\alpha \dot{x}+\beta x-\gamma \dot{x} \varepsilon_{0}-\frac{Q \gamma \dot{x} \varepsilon_{0}}{R T}-x \gamma \dot{x} \varepsilon_{0}-\frac{Q x \gamma \dot{x} \varepsilon_{0}}{R T}-\frac{1}{3} x^{2} \gamma \dot{x} \varepsilon_{0}- \\
&-\frac{Q x^{2} \gamma \dot{x} \varepsilon_{0}}{3 R T}-\frac{1}{6} x^{3} \gamma \dot{x} \varepsilon_{0}-\frac{Q x^{3} \gamma \dot{x} \varepsilon_{0}}{6 R T}=0 .
\end{aligned}
$$

\section{Method Of SOlution 1}

5.1. Homotopy perturbation method. Equation 13 is a nonlinear differential equation which is can be solved using HPM. To apply HPM consider the equation

$$
L(u)+N(u)=f(r) \quad r \in \Omega
$$

associated with the boundary conditions

$$
B\left(u, \frac{\partial u}{\partial n}\right)=0, \quad r \in \Gamma .
$$

Here $u(r)$ is the unknown, $L(u)$ and $N(u)$ are linear and nonlinear operators respectively, $f(r)$ is a known analytical function. $\Gamma$ is the boundary of the domain $\Omega$ with outward normal $n$.

The homotopy perturbation method proposed by Ji-Huan-He 11] can be expressed as

$$
H(u, p)=(1-p)\left[L(u)-L\left(u_{0}\right)\right]+p[L(u)+N(u)-f(r)]=0
$$

where $p$ is an embedding parameter and $u_{o}$ is an initial approximation of $u$. The solution is sought in the form

$$
u=u_{0}+p u_{1}+p^{2} u_{2}+p^{3} u_{3}+p^{4} u_{4}+p^{5} u_{5} \ldots
$$

In our case $t$ corresponds to $r, x$ to $u$ and the left side of 13 corresponds to $L(u)+$ $N(u)$ while $f(t)=0$. Hence

$$
\begin{aligned}
H(x, p)= & (1-p)\left(\frac{d^{2} x}{d t^{2}}-\frac{d^{2} x_{0}}{d t^{2}}\right)+ \\
& +p\left(\frac{d^{2} x}{d t^{2}}+\alpha \frac{d x}{d t}+\beta x-\gamma \varepsilon_{0} \frac{d x}{d t}\left(1+x+\frac{x^{2}}{3}+\frac{x^{3}}{6}\right)\left(1+\frac{Q}{R T}\right)\right)=0
\end{aligned}
$$

where

$$
x \simeq x_{0}+p x_{1}+p^{2} x_{2}+p^{3} x_{3}+p^{4} x_{4}+p^{5} x_{5}=\sum_{\ell=0}^{6} p^{\ell} x_{\ell} .
$$

Consequently

$$
\begin{array}{r}
H(x, p)=(1-p) \frac{d^{2}}{d t^{2}} \sum_{\ell=0}^{6} p^{\ell} x_{\ell}+p\left\{\frac{d^{2}}{d t^{2}} \sum_{\ell=0}^{6} p^{\ell} x_{\ell}+\alpha \frac{d}{d t} \sum_{\ell=0}^{6} p^{\ell} x_{\ell}+\beta \sum_{\ell=0}^{6} p^{\ell} x_{\ell}-\right. \\
\left.-\gamma \varepsilon_{0}\left(\frac{d}{d t} \sum_{\ell=0}^{6} p^{\ell} x_{\ell}\right)\left[1+\sum_{\ell=0}^{6} p^{\ell} x_{\ell}+\frac{1}{3}\left(\sum_{\ell=0}^{6} p^{\ell} x_{\ell}\right)^{2}+\frac{1}{6}\left(\sum_{\ell=0}^{6} p^{\ell} x_{\ell}\right)^{3}\right]\left(1+\frac{Q}{R T}\right)\right\}= \\
=0 .
\end{array}
$$


By making the coefficients of $p^{\ell}(\ell=0,1, \ldots, 5)$ equal to zero we can obtain the solutions for $x_{\ell}$. Expanding and resolving equation (21) the coefficients of $p^{\ell}$ are as follows:

$$
p^{0} ; \quad \frac{d^{2} x_{0}}{d t^{2}}=0,
$$

$p^{1} ; \quad \beta x_{0}+\alpha \frac{d x_{0}}{d t}-\left(1+\frac{Q}{R T}\right) \gamma \varepsilon_{0}\left[\frac{d x_{0}}{d t}+x_{0} \frac{d x_{0}}{d t}+\frac{1}{2} x_{0}^{2} x_{1} \frac{d x_{0}}{d t}+\frac{1}{6} x_{0}^{3} \frac{d x_{0}}{d t}\right]+$

$$
+\frac{d^{2} x_{0}}{d t^{2}}+\frac{d^{2} x_{1}}{d t^{2}}=0
$$

$p^{2} ; \quad \beta x_{1}+\alpha \frac{d x_{1}}{d t}+\frac{d^{2} x_{2}}{d t^{2}}-\left(1+\frac{Q}{R T}\right) \gamma \varepsilon_{0}\left(x_{1} \frac{d x_{0}}{d t}+x_{0} x_{1} \frac{d x_{0}}{d t}+\frac{1}{2} x_{0}^{2} x_{1} \frac{d x_{0}}{d t}+\right.$

$$
\left.\frac{d x_{1}}{d t}+x_{0} \frac{d x_{1}}{d t}+\frac{1}{2} x_{0}^{2} \frac{d x_{1}}{d t}+\frac{1}{6} x_{0}^{3} \frac{d x_{1}}{d t}\right)=0
$$

$p^{3} ; \quad \beta x_{2}+\alpha \frac{d x_{2}}{d t}+\frac{d^{2} x_{3}}{d t^{2}}+\alpha \frac{d x_{3}}{d t}-\left(1+\frac{Q}{R T}\right) \gamma \varepsilon_{0}\left(\frac{1}{2} x_{1}^{2} \frac{d x_{0}}{d t}+\frac{1}{2} x_{0} x_{1}^{2} \frac{d x_{0}}{d t}+x_{2} \frac{d x_{0}}{d t}+\right.$

$$
\begin{array}{r}
+x_{0} x_{2} \frac{d x_{0}}{d t}+\frac{1}{2} x_{0}^{2} x_{2} \frac{d x_{0}}{d t}+x_{1} \frac{d x_{1}}{d t}+x_{0} x_{1} \frac{d x_{1}}{d t}+\frac{1}{2} x_{0}^{2} x_{1} \frac{d x_{1}}{d t}+ \\
\left.+\frac{d x_{2}}{d t}+x_{0} \frac{d x_{2}}{d t}+\frac{1}{2} x_{0}^{2} \frac{d x_{2}}{d t}+\frac{1}{6} x_{0}^{3} \frac{d x_{2}}{d t}\right)=0,
\end{array}
$$

$p^{4} ; \quad \beta x_{3}+\frac{d^{2} x_{4}}{d t^{2}}-\left(1+\frac{Q}{R T}\right) \gamma \varepsilon_{0}\left(\frac{1}{6} x_{1}^{3} \frac{d x_{0}}{d t}+x_{1} x_{2} \frac{d x_{0}}{d t}+x_{0} x_{1} x_{2} \frac{d x_{0}}{d t}+\right.$

$$
\begin{aligned}
& +x_{3} \frac{d x_{0}}{d t}+x_{0} x_{3} \frac{d x_{0}}{d t}+\frac{1}{2} x_{0}^{2} x_{3} \frac{d x_{0}}{d t}+\frac{1}{2} x_{1}^{2} \frac{d x_{1}}{d t}+\frac{1}{2} x_{0} x_{1}^{2} \frac{d x_{1}}{d t}+ \\
& +x_{2} \frac{d x_{1}}{d t}+x_{0} x_{2} \frac{d x_{1}}{d t}+\frac{1}{2} x_{0}^{2} x_{2} \frac{d x_{1}}{d t}+x_{1} \frac{d x_{2}}{d t}+x_{0} x_{1} \frac{d x_{2}}{d t}+ \\
& \left.+\frac{1}{2} x_{0}^{2} x_{1} \frac{d x_{2}}{d t}+\frac{d x_{3}}{d t}+x_{0} \frac{d x_{3}}{d t}+\frac{1}{2} x_{0}^{2} \frac{d^{2} x_{3}}{d t^{2}}+\frac{1}{6} x_{0}^{3} \frac{d x_{3}}{d t}\right)=0 .
\end{aligned}
$$

The boundary conditions associated with the solution are as follows:

$$
x_{0}(0)=x_{1}(0)=\ldots=x_{4}(0)=0 .
$$

Making use of equations (22)- 26) and the initial conditions 27) the following solutions are obtained for $x_{\ell}(\ell=1, \ldots, 4)$ :

$$
\begin{gathered}
x_{0}=\lg \left(\varepsilon_{0}-\frac{\varepsilon_{i}}{\varepsilon_{0}}\right), \\
x_{1}=-t^{2} \beta x_{0} / 2, \\
x_{2}=\frac{1}{72 R T} t^{3} \beta x_{0}\left[3 R T(4 \alpha+t \beta)-2 \gamma \varepsilon_{0}(Q+R T)\left(6+6 x_{0}+3 x_{0}^{2}+x_{0}^{3}\right)\right], \\
x_{3}=\frac{1}{4320 R^{2} T^{2}} t^{4} \beta x_{0} \times
\end{gathered}
$$




$$
\begin{aligned}
& \left\{6 R^{2} t^{2} T^{2} \beta^{2}+5\left[-6 R T \alpha+(Q+R T) \gamma\left(6+6 x_{0}+3 x_{0}{ }^{2}+x_{0}{ }^{3}\right) \varepsilon_{0}\right]^{2}+\right. \\
& \left.+6 R t T \beta\left[12 R T \alpha-(Q+R T) \gamma\left(12+30 x_{0}+24 x_{0}{ }^{2}+11 x_{0}{ }^{3}\right) \varepsilon_{0}\right]\right\}, \\
& x_{4}=\frac{t^{5} \beta x_{0}}{362880}\left\{9 R^{3} t^{3} T^{3} \beta^{3}+14\left(6 R T \alpha-(Q+R T) \gamma \varepsilon_{0}\left(6+6 x_{0}+3 x_{0}^{2}+x_{0}^{3}\right)\right)^{3}+\right. \\
& +36 R^{2} t^{2} T^{2} \beta^{2}\left[6 R T \alpha-(Q+R T) \gamma \varepsilon_{0}\left(6+42 x_{0}+69 x_{0}^{2}+49 x_{0}^{3}\right)\right]+ \\
& +42 R t T \beta\left[36 R^{2} T^{2} \alpha^{2}-6 R T(Q+R T) \alpha \gamma \varepsilon_{0}\left(12+38 x_{0}+32 x_{0}^{2}+15 x_{0}^{3}\right)+\right. \\
& \left.\left.+(Q+R T)^{2} \gamma^{2} \varepsilon_{0}^{2}\left(36+228 x_{0}+384 x_{0}^{2}+360 x_{0}^{3}+203 x_{0}^{4}+71 x_{0}^{5}+14 x_{0}^{6}\right)\right]\right\}
\end{aligned}
$$

With $x_{0}, x_{1}, x_{3}, x_{4}$ and $x_{5}-x_{5}$ is not presented here - the solution for $x$ is

$$
x(t) \simeq \sum_{\ell=0}^{5} x_{\ell}(t)
$$

It follows on the basis of the strain equation (8) that

$$
\varepsilon(t) \simeq \varepsilon_{0}^{2}-\varepsilon_{0} e^{x} .
$$

If $\varepsilon_{0}=1$ we have

$$
\varepsilon(t) \simeq 1-e^{x}
$$

or

$$
\varepsilon(t) \simeq 1-e^{\sum_{\ell=0}^{5} x_{\ell}(t)} .
$$

\section{Method of SOlution 2}

6.1. Differential transforms method. The second method applied to solving the nonlinear differential equation $(13)$ is called DTM. The fundamental definitions and the operational characteristics of the method developed by Zhou in 1986 [16, 17] are given below.

Let $u(t)$ be an analytic function differentiable continuously in the domain $T$. Then $u(t)$ is differentiable continuously with respect to time $t$ :

$$
\frac{d^{p} u(t)}{d t^{p}}=\varphi(t, p) \quad \forall t \in T
$$

For $t=t_{i}$ it holds that $\varphi(t, k)=\varphi\left(t_{i}, k\right)$, where $k$ is a non-negative integer. Equation (37) can, therefore, be rewritten as

$$
U(k)=\varphi\left(t_{i}, k\right)=\left.\frac{d^{k} u(t)}{d t^{k}}\right|_{t=t_{i}}
$$

where $U(k)$ is called the spectrum of $u(t)$ at $t=t_{i}$. Using Taylor series $u(t)$ can be expressed as

$$
u(t)=\sum_{k=0}^{\infty} \frac{\left(t-t_{i}\right)^{k}}{k !} U(k)
$$


Equation 39 is called the inverse of $U(k)$. The differential transform of $u(t)$ is defined by the equation

$$
U(k)=\left.\frac{H^{k}}{k !} \frac{d^{k} u(t)}{d t^{k}}\right|_{t=0} \quad k=0,1,2, \ldots
$$

in which $U(k)$ represents the transformed function and $u(t)$ is the original function. The function $U(k)$ is confined in the interval $t \in[0, H]$ where $H$ is a given constant number. The differential inverse transformation of $U(k)$ is defined as

$$
u(t)=\sum_{k=0}^{\infty}\left(\frac{t}{H}\right)^{k} U(k) .
$$

6.2. The operational properties of the differential transformation method. Let $u(t)$ and $v(t)$ be the functions of $t$. The transformed functions are denoted by $U(k)$ and $V(k)$. It can be shown by using the fundamental mathematical operations of DTM that for instance

$$
\begin{aligned}
& \text { i. If } z(t)=\frac{d u(t)}{d t} \text { then } Z(k)=(k-1) U(k+1) ; \\
& \text { ii. If } z(t)=u(t) v(t) \text { then } Z(t)=\sum_{r=0}^{k} U(r) V(k-r) \quad r=0,1,2, \ldots ; \\
& \text { iii. If } z(t)=u^{m}(t) \text { then } \mathrm{Z}(\mathrm{t})=\sum_{\mathrm{r}=0}^{\mathrm{k}} \mathrm{U}^{\mathrm{m}-1}(\mathrm{r}) \mathrm{U}(\mathrm{k}-\mathrm{r}) \quad \mathrm{m}=1,2,3, \ldots
\end{aligned}
$$

6.3. DTM Solution Procedures. Hence, the differential transformation of equations (8) and (13) yields the following recursive equation:

$$
\begin{aligned}
x_{k+2}=-\frac{1}{(k+1)(k+2)}\left\{\alpha(k+1) x_{k+1}+\beta x_{k}-\ddots\right. \\
-y \varepsilon_{o}\left(1+\frac{Q}{R T}\right)\left[(k+1) x_{k+1}+\sum_{l=0}^{k}(l+1) x_{l+1} x_{k-l}+\right. \\
+\frac{1}{3} \sum_{p=0}^{k}\left(\sum_{l=0}^{p}(l+1) x_{l+1} x_{p-l} x_{k-p}\right)+ \\
\left.\left.+\frac{1}{6} \sum_{m=0}^{k}\left(\sum_{p=0}^{m}\left(\sum_{l=0}^{p}(l+1) x_{l+1} x_{p-l} x_{m-p} x_{k-m}\right)\right)\right]\right\}
\end{aligned}
$$

The initial conditions are of the form:

$$
x_{0}=\ln \left(\frac{\varepsilon_{o}^{2}-\varepsilon}{\varepsilon_{o}}\right) \quad x_{1}=0 .
$$

Assuming that $k=2,3,4,5$ we have solved equation (41) for $x_{2}, x_{3}, x_{4}$ and $x_{5}$ - here the results are presented for $x_{2}, x_{3}$ and $x_{4}$ only:

$$
\begin{gathered}
x_{2}=-\beta x_{0} / 2, \\
x_{3}=\frac{1}{6} \alpha \beta x_{0}+\frac{1}{6} y \varepsilon_{o} \beta\left(1+\frac{Q}{R T}\right)\left(x_{0}+x_{0}^{2}+\frac{1}{3} x_{0}^{3}+\frac{1}{d 6} x_{0}^{4}\right),
\end{gathered}
$$




$$
\begin{aligned}
x_{4}=-\frac{1}{4} \alpha\left[\frac{1}{6} \alpha \beta x_{0}+\frac{1}{6} y \varepsilon_{o} \beta\left(1-\frac{Q}{R T}\right)\left(x_{0}+x_{0}^{2}+\frac{1}{3} x_{0}^{3}+\frac{1}{6} x_{0}^{4}\right)\right]+ \\
+\frac{1}{24} \beta^{2} x_{0}+\frac{1}{12} y \varepsilon_{o}\left(1+\frac{Q}{R T}\right)-\frac{1}{2} y \varepsilon_{o} \beta\left(1+\frac{Q}{R T}\right)\left(x_{0}+x_{0}^{2}+\frac{1}{3} x_{0}^{3}+\frac{1}{6} x_{0}^{4}\right)+ \\
+3\left(\frac{1}{6} \alpha \beta x_{0}-\frac{1}{6} y \varepsilon_{o} \beta x_{0}\left(1+\frac{Q}{R T}\right)\left(x_{0}+x_{0}^{2}+\frac{1}{3} x_{0}^{3}+\frac{1}{6} x_{0}^{4}\right)\right)+ \\
+\left(\frac{1}{6} \alpha \beta x_{0}-\frac{1}{6} y \varepsilon_{o} \beta x_{0}^{2}\left(1+\frac{Q}{R T}\right)\left(x_{0}+x_{0}^{2}+\frac{1}{3} x_{0}^{3}+\frac{1}{6} x_{0}^{4}\right)\right)+ \\
\quad+\frac{1}{2}\left(\frac{1}{6} \alpha \beta x_{0}-\frac{1}{6} y \varepsilon_{o} \beta x_{0}^{3}\left(1+\frac{Q}{R T}\right)\left(x_{0}+x_{0}^{2}+\frac{1}{3} x_{0}^{3}+\frac{1}{6} x_{0}^{4}\right)\right)
\end{aligned}
$$

With $x_{\ell}$ the solution is of the form

$$
x(t) \simeq \sum_{\ell=0}^{5} x_{\ell} t^{\ell}
$$

while the strain function is given by

$$
\varepsilon(t)=\varepsilon_{o}^{2}-\varepsilon_{o} e^{x(t)}
$$

from where by substituting 45 we have

$$
\varepsilon(t) \simeq \varepsilon_{o}^{2}-\varepsilon_{o} e^{\sum_{\ell=0}^{5} x_{\ell} t^{\ell}}
$$

\section{Results And Discussion}

The results obtained by applying the homotopy perturbation and differential transform methods to the analysis of the problem of temperature effects on relaxation of creep strain in polymeric biomaterials are presented in Table 1. In order to validate these results, the fourth order Runge-Kutta numerical method is applied to solve the same problem. The results are compared and shown in Table 1. This comparison is also shown in Figures $2 \mathrm{a}$ and b. For Figure $2 \mathrm{a}$, the HPM solution is obtained by using four iterative terms $(n=4)$ while for Figure $2 \mathrm{p}$ the number of iterative terms is increased to twelve $(n=12)$. This shows that the higher the number of terms in the HPM model solution, the more accurate the solution will be. An error analysis shows that minimal error exists in the solutions obtained with the methods DTM and HPM.

The general description of how the material responds with an initial strain of 0.9 is also shown in Figure 2 The biomaterial does not exhibit an instant response to loading, but rather undergoes relaxation before it begins to respond to loading. In addition, as the temperature increases, the relaxation time increases, and the deformation increases proportionally at different temperatures. 
Table 1. Comparison of HPM, DTM and numerical method results

\begin{tabular}{|c|c|c|c|c|c|c|}
\hline \multicolumn{6}{|c|}{$\begin{array}{c}\text { Results obtained by the use of homotopy perturbation method if } \\
\varepsilon_{i}=0.9, \varepsilon_{0}=1, \alpha=2, \beta=0.5, \gamma=0.9, Q=5000 \mathrm{~J} / \mathrm{mol}, R=8.314 \mathrm{~J} / \mathrm{molK}, \mathrm{K}=300 K\end{array}$} \\
\hline $\begin{array}{c}\text { Time } \\
t\end{array}$ & $\begin{array}{c}\mathrm{HPM} \\
(n=4) \\
\text { Solution }\end{array}$ & $\begin{array}{c}\mathrm{HPM}_{2} \\
(n=12) \\
\text { Solution }\end{array}$ & $\begin{array}{c}\text { DTM } \\
\text { Solution }\end{array}$ & $\begin{array}{c}\text { Numerical } \\
\text { Solution } \\
\text { (RK4) }\end{array}$ & $\begin{array}{c}\text { Error } \\
\text { Difference } \\
\text { DTM-RK4 }\end{array}$ & $\begin{array}{c}\text { Error } \\
\text { Difference } \\
\text { HPM }_{2} \text {-RK4 }\end{array}$ \\
\cline { 2 - 7 } & \multicolumn{5}{|c|}{ Strain $\varepsilon(t)$} & \\
\hline \hline 0.00 & 0.9000000 & 0.9000000 & 0.9000000 & 0.9000000 & 0.00000000 & 0.00000000 \\
\hline 0.10 & 0.8995268 & 0.8995267 & 0.8995267 & 0.8995267 & 0.00000000 & 0.00000000 \\
\hline 0.20 & 0.8984212 & 0.8984150 & 0.8984150 & 0.8984150 & 0.00000000 & 0.00000000 \\
\hline 0.30 & 0.8970945 & 0.8970225 & 0.8970225 & 0.8970225 & 0.00000000 & 0.00000000 \\
\hline 0.40 & 0.8961919 & 0.8957820 & 0.8957820 & 0.8957820 & 0.00000000 & 0.00000000 \\
\hline 0.50 & 0.8970340 & 0.8954685 & 0.8954685 & 0.8954685 & 0.00000000 & 0.00000000 \\
\hline 0.60 & 0.9019594 & 0.8974244 & 0.8974244 & 0.8974244 & 0.00000000 & 0.00000000 \\
\hline 0.70 & 0.9139977 & 0.9035709 & 0.9035709 & 0.9035709 & 0.00000000 & 0.00000000 \\
\hline 0.80 & 0.9348554 & 0.9158586 & 0.9158586 & 0.9158586 & 0.00000000 & 0.00000000 \\
\hline 0.90 & 0.9612801 & 0.9348325 & 0.9348325 & 0.9348325 & 0.00000000 & 0.00000000 \\
\hline 1.00 & 0.9842194 & 0.9578233 & 0.9578233 & 0.9578233 & 0.00000000 & 0.00000000 \\
\hline 1.10 & 0.9963269 & 0.9789104 & 0.9789104 & 0.9789104 & 0.00000000 & 0.00000000 \\
\hline 1.20 & 0.9996162 & 0.9926209 & 0.9926209 & 0.9926209 & 0.00000000 & 0.00000000 \\
\hline 1.30 & 0.9999868 & 0.9984012 & 0.9984012 & 0.9984012 & 0.00000000 & 0.00000000 \\
\hline 1.40 & 0.9999999 & 0.9998149 & 0.9998149 & 0.9998149 & 0.00000000 & 0.00000000 \\
\hline 1.50 & 1.0000000 & 0.9999904 & 0.9999904 & 0.9999904 & 0.00000000 & 0.00000000 \\
\hline 1.60 & 1.0000000 & 0.9999998 & 0.9999998 & 0.9999998 & 0.00000000 & 0.00000000 \\
\hline 1.70 & 1.0000000 & 1.0000000 & 1.0000000 & 1.0000000 & 0.00000000 & 0.00000000 \\
\hline 1.80 & 1.0000000 & 1.0000000 & 1.0000000 & 1.0000000 & 0.00000000 & 0.00000000 \\
\hline 1.90 & 1.0000000 & 1.0000000 & 1.0000000 & 1.0000000 & 0.00000000 & 0.00000000 \\
\hline 2.00 & 1.0000000 & 1.0000000 & 1.0000000 & 1.0000000 & 0.00000000 & 0.00000000 \\
\hline
\end{tabular}

Thermal coefficient at varying temperatures. The response of the biomaterial to varying thermal coefficients is shown in Figure 2. It is observed that an increase in the thermal coefficient resulted in a decrease in the deformation and an increase in the rate of relaxation in the material. It is also observed that the material shows a high resistance to deformation at high thermal coefficient.

The effect of four thermal conductivity coefficients $\gamma$ at different temperatures of the material model is shown in Figure 4. The differences among the responses at these different temperatures are minimal. Material exhibits longer relaxation before it begins to deform at lower thermal conductivity coefficients than at higher thermal conductivity coefficients. There is sharp increase in deformation at higher thermal conductivity coefficients. 

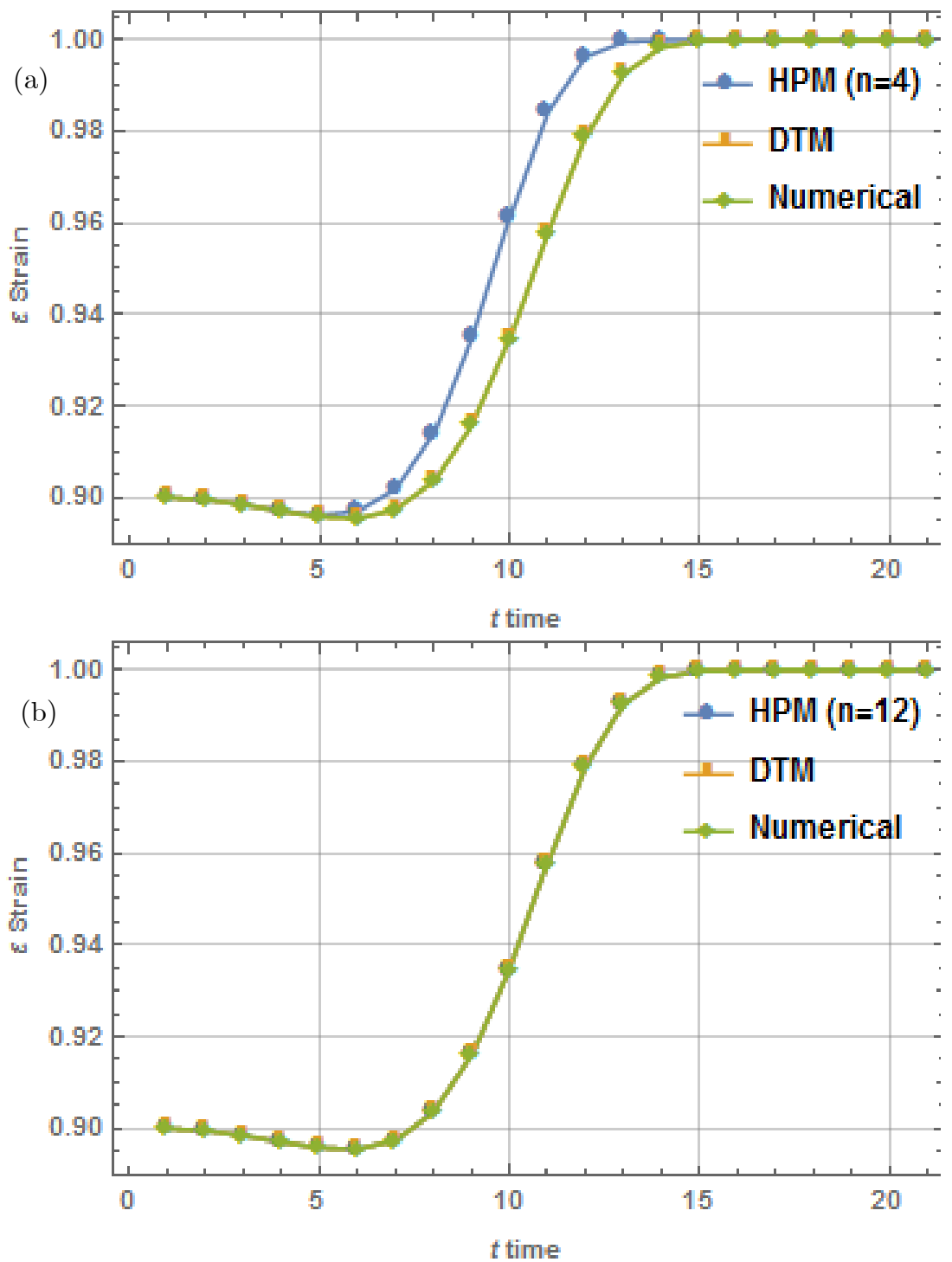

Figure 2. Comparison of the numerical results obtained by using the three methods; DTM, HPM and the fourth order Runge-Kutta $\operatorname{method}\left(\varepsilon_{i}=0.9, \varepsilon_{0}=1, \alpha=2, \beta=0.5, \gamma=0.9, Q=5000 \mathrm{~J} / \mathrm{mol}\right.$, $R=8.314 \mathrm{~J} / \mathrm{mol} \mathrm{K}, T=300 \mathrm{~K}$ ); (a) $n=4$, (b) $n=12$ 

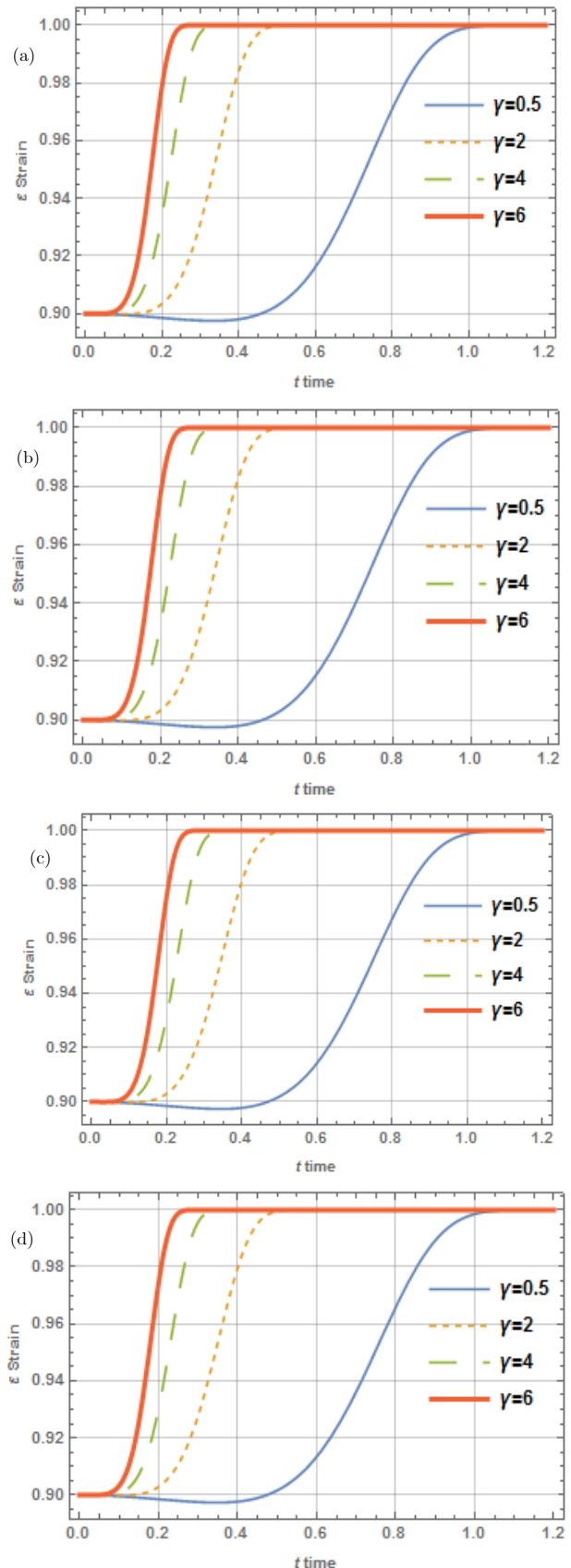

Figure 3. Variation of thermal coefficients at different temperatures $\left(\varepsilon_{i}=0.9, \varepsilon_{0}=1, Q=50000 \mathrm{~J} / \mathrm{mol}, R=8.314 \mathrm{~J} / \mathrm{mol} \mathrm{K}, \alpha=0.5, \beta=0.5\right.$, (a) $T=300 \mathrm{~K}$, (b) $T=305 K$, (c) $T=310 \mathrm{~K}$, (d) $T=315 \mathrm{~K}$ ) 

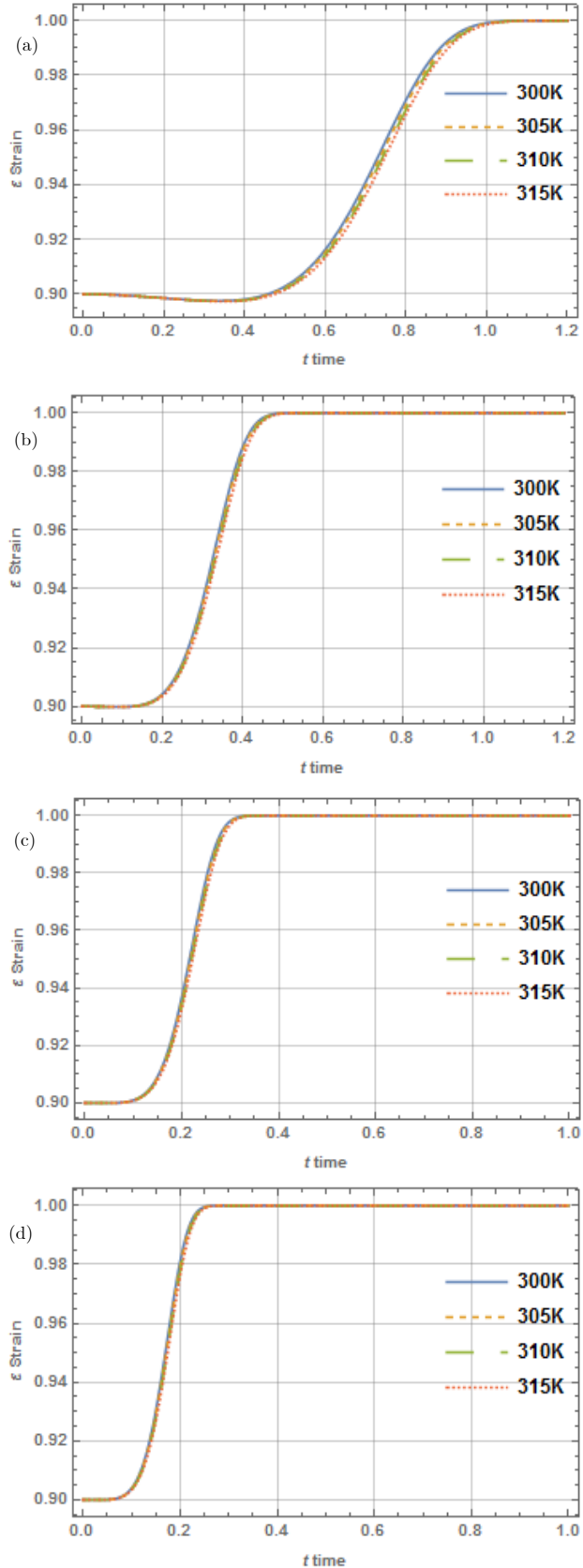

Figure 4. Temperature variation with thermal conductivity $\left(\varepsilon_{i}=0.9\right.$, $\varepsilon_{0}=1, Q=50000 \mathrm{~J} / \mathrm{mol}, R=8.314 \mathrm{~J} / \mathrm{mol} \mathrm{K}, \alpha=2, \beta=0.5$, (a) $\gamma=0.5$, (b) $\gamma=2$, (c) $\gamma=4$, (d) $\gamma=6$ ) 

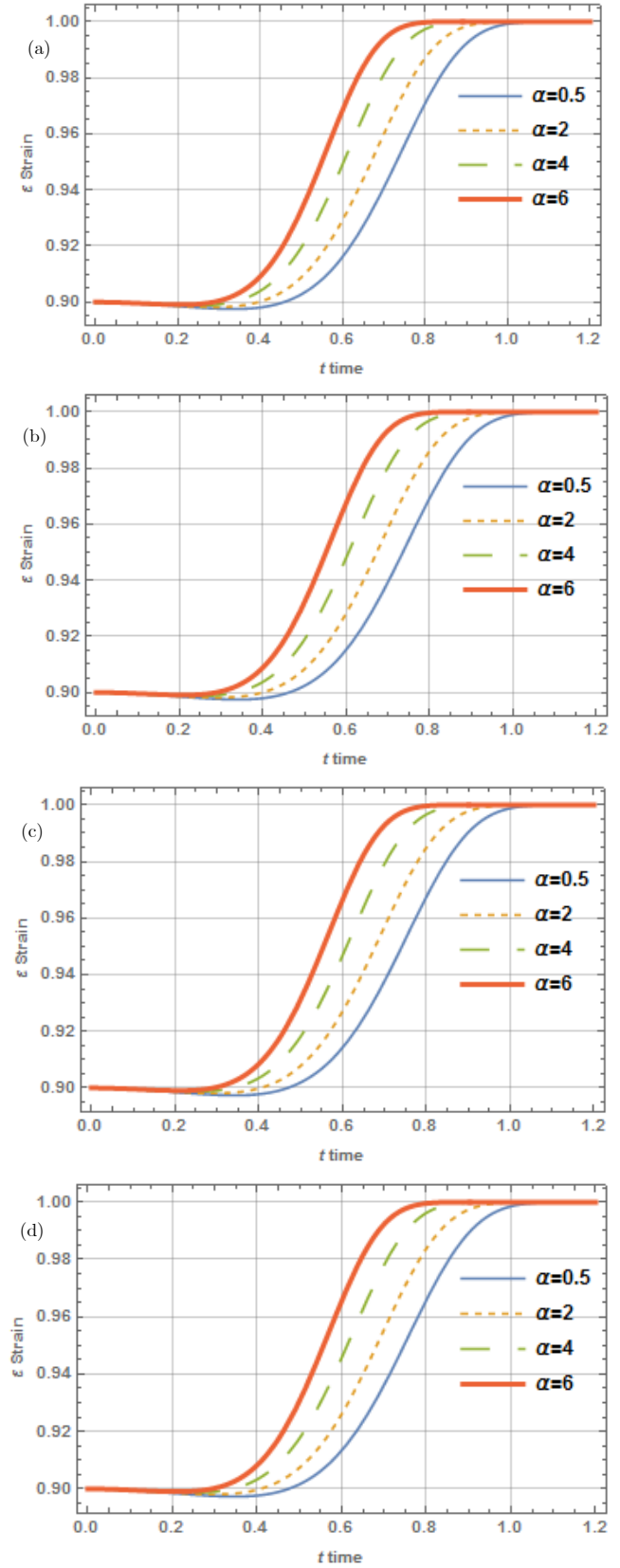

Figure 5. The effects of the viscosity coefficient on the developed model $\left(\varepsilon_{i}=0.9, \varepsilon_{0}=1, Q=50000 \mathrm{~J} / \mathrm{mol}, R=8.314 \mathrm{~J} / \mathrm{mol} \mathrm{K}, \alpha=2\right.$, $\beta=0.5, \gamma=0.5$, (a) $T=300 \mathrm{~K}$, (b) $T=305 \mathrm{~K}$, (c) $T=310 K$, (d) $T=315 \mathrm{~K})$ 

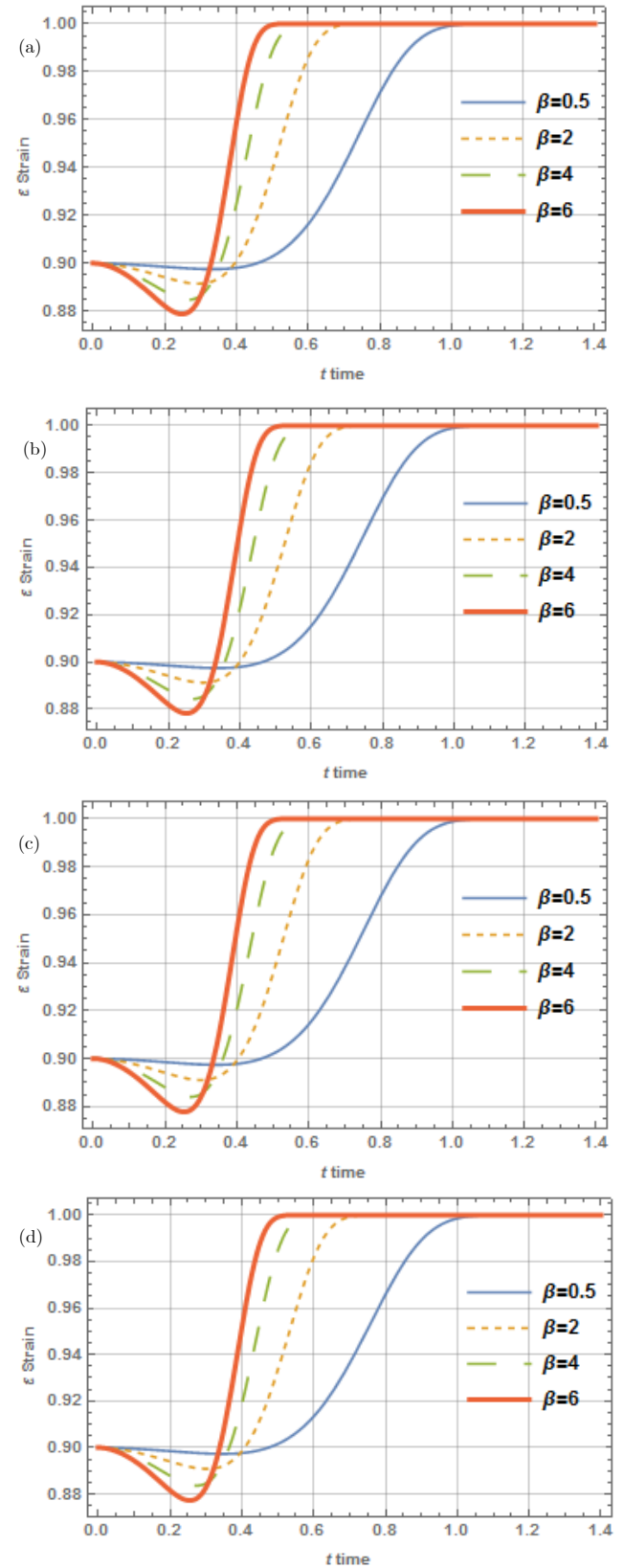

Figure 6. The effects of the stiffness coefficient on the developed model $\left(\varepsilon_{i}=0.9, \varepsilon_{0}=1, Q=50000 \mathrm{~J} / \mathrm{mol}, R=8.314 \mathrm{~J} / \mathrm{molK}, \alpha=2\right.$, $\beta=0.5, \gamma=0.5$, (a) $T=300 K$, (b) $T=305 K$, (c) $T=310 K$, (d) $T=315 K)$ 
Viscosity coefficient at varying temperatures. Figure 5 show the response of a material at a varying viscosity coefficient $\alpha$ of $0.5,2,4$ and 6 . As the viscosity coefficient increases, the difference in the deformation path between the high and low temperature decreases. It is also observed that at a relatively low viscosity, material tends to deform more compared to when it is high, i.e. there is an increase in resistance to deformation as the viscosity coefficient increases. Similarly, at low viscosity, the relaxation period takes a longer time before the material begins to deform.

Stiffness coefficient at varying temperatures. The response of the material to different stiffness coefficients $\beta$ is shown in Figure 6. It is observed that the higher the stiffness coefficient, the shorter time the material takes to deform. The relaxation time was shorter for a material with higher stiffness and lower temperature, while materials with lower stiffness as shown in Figure 6a tend to show higher resistance to deformation than those with a lower stiffness coefficient.

\section{Conclusion}

In this study, the analytical solution of the effects of temperature on relaxation of creep strain in polymeric biomaterials governed by a developed nonlinear differential equation has been presented. The developed model was solved using two efficient methods; the homotopy perturbation method and differential transform method. The obtained results were validated with the fourth-order Runge-Kutta numerical method and the error analysis showed good agreement among the results. The effects of thermal conductivity, viscosity and material stiffness on the deformation of these biomaterials were investigated. As the thermal conductivity and viscosity of the material increase, its resistance to deformation increases, while an increase in the material stiffness resulted in an increase in the rate of deformation and relaxation. The analytical solution developed in this work provides a better understanding of the relationship between the physical quantities of the problem investigated. The results obtained in this theoretical investigation will assist in the analysis of the temperature effects on relaxation of creep strain in biomaterials and in the handling of various parameters in the developed model.

\section{REFERENCES}

1. I. D. Ibrahim, E. R. Sadiku, T. Jamiru, A. Hamam, and W. K. Kupolati. "Applications of polymers in the biomedical field." Curremt Trends Biomedical Engineering Bioscience, 4, (2017), pp. 9-11. DOI: 10.19080/CTBEB .2017.04.555650.

2. J. L. Drury, R. G. Dennis, and D. J. Mooney. "The tensile properties of alginate hydrogels." Biomaterials, 25, (2004), 3187-3199. DoI: 10.1016/j.biomaterials. 2003.10.002.

3. J. B. Park and R. S. Lakes. "Structure - Property Relationships of Biological Materials." Biomaterials, An Introduction, Springer, 1992. Chap. 9, pp. 185222. DOI: $10.1007 / 978-1-4684-3423-1$

4. F. H. Silver, J. W. Freeman, and G. Bradica. "Orthopedic Biology and Medicine." Repair and Regeneration of Ligaments, Tendons, and Joint Capsule. Ed. by William R. Walsh. Springer, 1992. Chap. 1, pp. 15-47. DOI: 10.1385/1592599427. 
5. M. D. Monsia. "A mathematical model for predicting the relaxation of creep strains in materials." Physical Review \&6 Research International, 2(3), (2012), pp. $107-124$.

6. R. H. Ewoldt, A. E. Hosoi, and G. H. McKinley. "Nonlinear viscoelastic biomaterials: meaningful characterization and engineering inspiration." Integrative and Comparative Biology, 49(1), (2009), pp. 40-50. DOI: 10.1093/icb/icp010.

7. B. K. Appelt. "Advanced Substrates: A Materials and Processing Perspective." Materials for Advanced Packaging, ed. by Lu D. and Wong C. Springer, Cham, 2017, pp. 287-330. DOI: 10.1007/978-3-319-45098-8_7.

8. L. M. Lazaro and D. A. G. Aranda. "Process temperature profile and rheological properties of greases from vegetable oils." Green and Sustainable Chemistry, 04(1), (2014), pp. 38-43. DOI: 10.4236/gsc.2014.41007

9. A. Hernandez-Jimenez, J. Hernandez-Santiago, A. Macias-Carcia, and J. SanchezGonzalez. "Relaxation modulus in PMMA and PTFE fitting by fractional Maxwell model." Polymer Testing, 21(3), (2014), pp. 325-331. DOI: $10.1016 /$ S01429418(01)00092-7.

10. M. Abu-Abdeen. "Single and double-step stress relaxation and constitutive modeling of viscoelastic behavior of swelled and un-swelled natural rubber loaded with carbon black." Materials and Design, 31(4), (2010), 2078-2084. DOI: 10. 1016/j.matdes.2009.10.006.

11. J. H. He. "Homotopy perturbation method: A new nonlinear analytical technique." Applied Mathematics and Computation, 135(1), (2003), pp. 73-79. DOI: 10.1016/S0096-3003(01)00312-5.

12. T. Öziş and A. Yıldırım. "Homotopy perturbation method: A new nonlinear analytical technique." Computers $\&$ Mathematics with Applications, 56(5), (2008), pp. 1216-1224. DOI: $10.1016 / \mathrm{j}$. camwa.2008.02.023.

13. S. Saedodin and M. Shahbabaei. "Thermal analysis of natural convection in porous fins with homotopy perturbation method (HPM)." Arabian Journal for Science and Engineering, 38(8), (2013), 2227-2231. DOI: 10.1007/s13369-0130581-6.

14. H. A. Hoshyar, I. Rahimipetroudi, D. D. Ganji, and A. R. Majidian. "Thermal performance of porous fins with temperature-dependent heat generation via homotopy perturbation method and collocation method." Journal of Applied Mathematics and Computational Mechanics, 14(4), (2015), pp. 53-65. DOI: 10.17512/jamcm.2015.4.06.

15. O. A. Adeleye and A. Yinusa. "Heat transfer analysis of non-newtonian natural convective fluid flow using homotopy perturbation and Daftardar-Gejiji and Jafari methods." Journal of Applied Mathematics and Computational Mechanics, 18(2), (2015), pp. 5-18. DOI: 10.17512/jamcm.2019.2.01.

16. J.K. Zhou. Differential Transformation and its Applications for Eectrical Circuits. Huazhong University Press, 1986.

17. Xi Chen, Jie Xiang Lou, and Ying Dai. "Differential transform method for the brooks - Corey model." Advances in Civil and Structural Engineering III, vol. 501. Applied Mechanics and Materials. Trans Tech Publications Ltd., Apr. 2014, pp. 2520-2523. DOI: 10.4028/www.scientific.net/AMM.501-504.2520 
18. T. M. Elzaki and E. M. A. Hilal. "Solution of linear and nonlinear partial differential equations using mixture of Elzaki transform and the projected differential transform method." Journal of Applied Mathematics and Computational Mechanics, 2(1), (2012), pp. 50-59.

19. M. Garg, P. Manohar, and S. L. Kalla. "Generalized differential transform method to space-time fractional telegraph equation." International Journal of Differential Equations, Article ID 548982, (2011), pp. 1-10. DOI: 10.1155/2011/548982.

20. O. O. Agboola, A. A. Opanuga, and J. A. Gbadeyan. "Solution of third order ordinary differential equations using differential transform method." Global Journal of Pure and Applied Mathematics, 11(4), (2015), pp. 2511-2517.

21. S. Mukherjee, D. Goswami, and B. Roy. "Solution of higher-order Abel equations by differential transform method." International Journal of Modern Physics C, 23(9), (2012), pp. 1250056-3. DOI: 10.1142/S0129183112500568.

22. C. Kuo, M. Fanton, L. Wu, and D. Camarillo. "Spinal constraint modulates head instantaneous center of rotation and dictates head angular motion." Journal of Biomechanics, 76, (2018), pp. 220-228. DOI: 10.1016/j · jbiomech.2018.05. 024

23. M. Thongmoon and S. Pusjuso. "The numerical solution of differential transform method and Laplace transform method for a system of differential equations." Nonlinear Analysis: Hybrid Systems, 4(3), (2018), pp. 425-431. DOI: 10.1016/ j.nahs.2009.10.006.

24. O. Adeleye, O. Olawale, and G. Sobamowo. "Prediction of phagocyte transmigration for foreign body responses to subcutaneous biomaterial implantations using differential transform method." Journal of Biomimetics, Biomaterials and Biomedical Engineering, 32(7), (2017), pp. 98-114. DOI: $10.4028 /$ www.scientific.net/JBBBE.32.98.

25. O. Adeleye, A. Eloka, and G. Sobamowo. "Prediction of creep strain relaxations in biomaterials using differential transformation method." Journal of Biomimetics, Biomaterials and Biomedical Engineering, 38(8), (2018), pp. 11-22. DOI: 10.4028/www.scientific.net/JBBBE.38.11.

26. R. D. Bauer. "Rheological approaches of arteries." Journal of Biomimetics, Biomaterials and Biomedical Engineering, 23(1), (1984), pp. 159-167. DOI: 10 . 3233/bir-1984-23s129.

27. G. Z. Quan, Y. Shi, Y. X. Wang, Kang B. S., Ku T. W., and Song W. J. "Constitutive modelling for the dynamic recrystallization evolution of AZ80 magnesium alloy based on stress-strain data." Journal of Biomimetics, Biomaterials and Biomedical Engineering, 528(28), (2011), pp. 8051-8059. DOI: 10.1016/j.msea.2011.07.064

28. C. X. Yue, L. W. Zhang, S. L. Liao, J. B. Pei, H. J. Gao, Y. W. Jia, and X. Lian. "Research on the dynamic recrystallization behaviour of Gcr15 steel." Materials Science and Engineering: A, 499(1-2), (2009), pp. 177-181. DOI: $10.1016 / \mathrm{j}$. msea.2007.11.123. 